\title{
LA PARTICIPACIÓN CIUDADANA EN LA SUPERACIÓN DE LA POBREZA. REALIDAD Y DESAFÍOS PARA LA DEMOCRACIA CHILENA
}

\author{
Gonzalo Delamaza \\ Universidad de Los Lagos, Chile \\ gonzalo.delamaza@ulagos.cl
}

\section{RESUMEN}

La participación ciudadana es un concepto polisémico cuya utilización política incrementa dicha característica. Más allá de la literatura que ensalza las virtudes de la participación, este artículo plantea que, en el caso de sociedades desiguales con amplios sectores excluidos y en situación de pobreza, clarificar el significado permite vincular participación y superación de la pobreza. Se postula que en dichos casos la participación contribuirá a la superación de la pobreza solo si involucra redistribución del poder, lo cual vuelve al concepto un objeto de disputa político relevante. Se proponen dimensiones conceptuales sobre la relación entre ambos procesos. Se analiza la normativa sobre participación en el caso chileno, estableciéndose numerosas limitaciones, así como algunos desafíos y propuestas para incorporar la participación ciudadana como componente de las políticas de superación de la pobreza en un marco de profundización de la democracia en el país.

Palabras clave: Participación, Pobreza, Políticas públicas, Derechos humanos, Chile. 


\title{
CITIZEN PARTICIPATION IN OVERCOMING POVERTY REALITY AND CHALLENGES FOR CHILEAN DEMOCRACY
}

\begin{abstract}
Citizen participation is a polysemic concept whose political use accentuates this characteristic. Looking beyond the literature that extols the virtues of participation, this article asserts that, in unequal societies with large excluded sectors in a situation of poverty, it is possible, by clarifying the concept's meaning, to link participation and the defeat of poverty. The article argues that, in these cases, participation will contribute to overcoming poverty only if it involves a redistribution of power, which makes the concept an object of important political dispute. The article addresses conceptual dimensions of the relationship between the two processes. It analyzes regulation of participation in the Chilean case, identifying numerous limitations, and examines some challenges and proposals for incorporating citizen participation as a component of policies to overcome poverty in a framework of the deepening of democracy in Chile.
\end{abstract}

Keywords: Participation, Poverty, Public policies, Human rights, Chile. 


\section{INTRODUCCIÓN}

El desafío de la participación forma parte hace ya algún tiempo del discurso de las políticas públicas. Aunque no ocupa un lugar protagónico, ni está en el primer lugar de las prioridades políticas, poco a poco se va instalando como parte de "lo que hay que hacer" en la materia. El caso chileno ha sido de incorporación tardía en relación a otros países de la región. En Colombia y Brasil este componente forma parte de los derechos constitucionales desde comienzos de los años noventa. Experiencias como los presupuestos participativos se han generalizado no solo en Brasil, sino también en ciudades europeas y en grandes capitales como Buenos Aires, Montevideo y Ciudad de México en diversos períodos, mientras en Perú se institucionalizó en el ámbito regional como una obligación legal y administrativa. Bolivia, Ecuador y también Venezuela han incorporado organismos de poder ciudadano a nivel local en el marco de proyectos de transformación política. Chile no ha dado aún ninguno de esos pasos, pero desde 2011 cuenta con la Ley 20.500 de Asociatividad y Participación Ciudadana en la Gestión Pública, operativa desde su reglamentación en 2012, que analizaremos en este trabajo.

Es posible periodizar el caso chileno del siguiente modo. A partir de 1990 se instala en la política social chilena el uso del apellido "participativo" para denominar varios de sus programas, ingresando de este modo en el léxico de las políticas públicas. Organismos como el Fondo de Solidaridad e Inversión Social (FOSIS), creado el mismo año 1990, enfatizaron en ese enfoque en sus programas de gestión local en contextos de pobreza. Igual cosa ocurrió con los Pavimentos Participativos, un programa del Ministerio de Obras Públicas (MOP) que existe hasta hoy. Luego de la pérdida de apoyo electoral de la coalición gobernante en diciembre de 1999 (1.000.000 de votos), la participación comienza a tratarse como un tema político, lo que conduce a la modificación de la Ley Orgánica Constitucional de Municipalidades ese mismo ańo, que consagra las audiencias públicas, las ordenanzas municipales de participación y los plebiscitos comunales de origen alcaldicio y ciudadano. En 2004 se presenta el proyecto de Ley de Participación y se ponen en práctica mecanismos administrativos en el ejecutivo central, como parte de las iniciativas de modernización del Estado. A partir de 2007 se percibe mayor dinamismo en la normativa: se aprueba la Ley de Transparencia y Acceso a la Información Pública, se ratifica el Convenio 169 de la OIT que consagra la consulta indígena y en 2011 se aprueba la Ley de Asociatividad y Participación Ciudadana en la Gestión Pública. 
Si en la primera etapa hubo un nexo entre participación local (cofinanciamiento y organización de la demanda local), en el segundo no es este el facto predominante. Como veremos, la participación institucionalizada tiende a reproducir la desigualdad y la marginación de los grupos pobres, con la excepción de la consulta indígena. En ambos períodos la participación está limitada a las modalidades consultivas o informativas, sin ingresar en el campo de la toma de decisiones. Tampoco se ponen en práctica mecanismos de democracia directa ${ }^{1}$.

Más allá de los enfoques normativos que enfatizan las diferentes virtudes que tendría una política pública más participativa, pero que difícilmente encontramos puestos en práctica, se puede establecer que incrementar la participación es una necesidad de la gobernanza contemporánea en sociedades complejas (Blanco y Gomá 2002) y forma parte de los desafío de construcción de una sociedad inclusiva, desafíos que no se satisfacen con las formas asistencialistas, paternalistas y clientelistas tradicionales en la relación entre las instituciones y sus autoridades con los grupos en situación de pobreza ${ }^{2}$.

Pero si bien esta necesidad comienza a ser reconocida incluso en contextos como el chileno, dominado por el enfoque tecnocrático y la concepción elitista de la política (Delamaza 2015), aún subsiste una gran ambigüedad en cuanto a su definición y alcances, que es necesario clarificar. Y, por otra parte, más allá del consenso aparente de que "todos queremos la participación", es necesario reconocer que también se trata de un asunto conflictivo, en torno al cual hay disputas legítimas que es necesario encarar. Por último, al incorporar un énfasis específico en la participación de los grupos pobres y excluidos, se está haciendo alusión directa a una necesidad de inclusión social tanto objetiva como subjetiva, es decir legítima. Nuestro punto de vista es que, en sociedades desiguales el desafío de participación se refiere principalmente a la incorporación de quienes casi nunca participan,

1 En un Índice de Democracia Directa aplicado a 18 países de América Latina, Chile resulta en penúltimo lugar. Solo El Salvador tiene menos mecanismos de democracia directa consagrados constitucionalmente (Lissidini 2015: 150).

2 Convengamos desde el inicio que las prácticas asistencialistas, paternalistas, corporativistas y clientelares no se superan automáticamente por el hecho de establecer mecanismos institucionales de participación ciudadana, puesto que pueden reproducirse también al interior de estos, del mismo modo que lo hacen a través de los mecanismos "tecnificados" de la política social como son los concursos de proyectos y la focalización (Durston 2012). El desafío de la participación incluye diseños adecuados, cambio cultural, priorización política y continuidad en las estrategias para tener impactos reales. 
lo cual supone una transferencia de poder que, sin duda, tiene implicancias políticas relevantes y, por lo tanto, es objeto de disputa. Por ello debemos analizar t el contexto histórico-político en el cual surgen los mecanismos de participación, pero, por sobre todo, establecer distinciones que permitan discriminar las características de estos mecanismos con el fin de establecer si pueden o no tener una mayor potencialidad de incluir y favorecer a los grupos pobres.

Este trabajo consta de cuatro secciones. En la primera de ellas se establece el diagnóstico de la situación actual de la participación en Chile, con énfasis en la que se practica entre y con los grupos en situación de pobreza. Allí se argumenta que, aun existiendo mecanismos de participación, su incidencia es escasa, lo que los vuelve irrelevantes. En la segunda parte se establecen elementos conceptuales para diferenciar tipos de participación y se propone una concepción de esta entendida como un derecho humano que debe ser garantizado. En la tercera parte se describen los avances normativos e institucionales de la participación en el país. Finalmente, se plantean algunos desafíos y propuestas para incorporar la participación ciudadana como componente de las políticas de superación de la pobreza en un marco de profundización de la democracia en Chile.

\section{BALANCE A TREINTA AÑOS DE LA RECUPERACIÓN DEMOCRÁTICA EN CHILE: ABUNDANCIA DE ACCIONES PARTICIPATIVAS CON POCA INCIDENCIA EN LA ORIENTACIÓN Y DECISIÓN DE LAS POLÍTICAS}

Aunque la participación como componente de la retórica política y como parte integrante de programas diversos tiene ya larga data, nuestro diagnóstico es que ella no ha incrementado su potencial transformador. En los programas orientados a la superación de la pobreza, la participación ha formado parte del discurso oficial. Más aun, podríamos seńalar que gran parte de los programas del área social contienen elementos participativos. Con la aprobación de la Ley 20.500 en 2011 se incorporaron incluso mecanismos institucionales obligatorios de participación en una multiplicidad de organismos públicos (Consejo Nacional de Participación Ciudadana y Fortalecimiento de la Sociedad Civil 2017). Para una gran cantidad de organismos de la rama ejecutiva se establecen los Consejos de la Sociedad Civil (consultivos), la exigencia de contar con una Norma de Participación y la realización de consultas ${ }^{3}$. En los municipios se crean los

3 También se incorporan mecanismos de acceso a la información, que aquí no consideramos propiamente de participación ciudadana, sino como precondiciones de ella. 
Consejos de Organizaciones de la Sociedad Civil (COSOC, consultivos), conformados con representantes de las organizaciones electos por las organizaciones registradas en el municipio, como un nuevo órgano de la gestión comunal ${ }^{4}$. Se crea también el Fondo de Fortalecimiento de Organismos de Interés Público, financiado con el presupuesto de la Nación.

Desde este punto de vista, el diagnóstico usual, sobre todo entre la dirigencia social, de que "faltan mecanismos de participación" no nos parece preciso, por varias razones que se detallan en el texto. El problema reside, a nuestro juicio, en el lugar subordinado y de baja significación que ocupa la participación ciudadana en el ciclo de las políticas públicas orientadas a la superación de la pobreza y, por lo tanto, el impacto poco relevante de los mecanismos que se utilizan. Postulamos que ello se debe a razones bastante profundas de carácter político e institucional y, por lo tanto, que la posibilidad de modificar esta situación está asociada a una concepción donde la participación sea considerada como un derecho a ser garantizado, así como a la realización de las reformas institucionales que así lo permitan.

Una primera consideración es de contexto político más general. Hemos planteado en otros trabajos que el "modelo de gobernabilidad" que ha orientado el funcionamiento del sistema político democrático en Chile, tenía como propósitos principales los siguientes: 1) garantizar la continuidad del crecimiento económico, basado en la explotación intensiva de los recursos naturales, lo que suponía la apertura al exterior por medio de la producción privada e implicaba una fuerte concentración económica, por la alta demanda de capital involucrada; 2) la estabilidad política, basada en la continuidad (y no en la ruptura constitucional), el elitismo democrático y el equilibrio de los dos grandes bloques políticos ${ }^{5}$ 3) la activación de las políticas sociales compensatorias destinadas a reducir la pobreza, a las que posteriormente se agregó el objetivo de protección social (Delamaza 2015). Esta última dimensión es muy relevante, puesto que la pobreza medida en términos de ingreso y luego de manera multidimensional disminuyó significativamente durante el período considerado y las políticas sociales fueron un factor importante para ello. Se mantuvo las políticas sociales

4 En el ámbito regional y provincial. la ley no introdujo mecanismos de participación.

5 El elitismo democrático es una concepción de la democracia que considera que el componente fundamental de la misma es la selección competitiva y pluralista de elites políticas organizadas en partidos. Fue desarrollada originalmente en Europa en el contexto posterior a la segunda guerra mundial, para reducir el riesgo de resurgimiento de expresiones como el fascismo y el nazismo (Avritzer 2002). 
focalizadas, aunque ampliando los focos respecto de lo hecho durante el gobierno militar, se incrementó significativamente el financiamiento de la infraestructura social y se estableció luego un sistema de protección social, que incluyó en su diseńo un enfoque de derechos garantizados (Robles 2013). También es relevante para este trabajo, pues en el ámbito social fue donde se pusieron en práctica los principales mecanismos de participación en programas específicos.

En ese diseño la participación sustantiva se canalizaba principalmente hacia el ámbito electoral en los marcos predefinidos. Cualquier otro mecanismo puesto en práctica no debía en ningún momento poner en juego aspectos relativos a las decisiones u orientaciones de política. Al contrario, el enfoque estaba basado en la subordinación política de las expresiones sociales y ciudadanas, las que debían encuadrarse en los marcos de la política social. Las políticas y programas de superación de la pobreza, siguieron, por lo tanto, esa orientación y definieron su tarea no frente a actores sociales constituidos como contrapartes, sino frente a lo que se denominó "la deuda social". El concepto mismo de pobreza ha transitado difícilmente desde una comprensión de pura carencia, a enfoques que incorporen el protagonismo de los sujetos sociales que se encuentran en dicha condición.

Se han implementado multiplicidad de programas que incluyen participación. En ellos predomina la participación de los involucrados directos en lo que denominamos "el último eslabón de la cadena", es decir, en la selección e implementación de proyectos comunitarios. No ocurre lo mismo en otras fases de la política como la formación de agenda, la formulación de políticas, el diseño, control o retroalimentación de los programas o la conformación y orientación de instituciones. Las formas organizativas empleadas tienden a ser ad hoc, fragmentadas y asociadas a intereses sectoriales, es decir responden a los intereses específicos de cada programa y segmentan a la comunidad de acuerdo a ellos (adultos mayores, jóvenes consumidores de drogas, pacientes de una determinada enfermedad). Esta modalidad redunda también en una falta de continuidad de los procesos participativos, pues están más asociados a los vaivenes de la implementación y a la voluntad política de determinadas autoridades, que a un derecho garantizado de las poblaciones ${ }^{6}$. Por otra parte, como los programas carecen de estrategias incrementales, de escalamiento e

6 En 2009, la Fundación para la Superación de la Pobreza propuso establecer una garantía a los usuarios de programas sociales en términos de que "tendrán asegurada su participación en dispositivos (comités de usuarios) que permitan recuperar sus opiniones y propuestas sobre el diseño, implementación y evaluación de los programas 
institucionalización, la discontinuidad se agudiza. La concursabilidad de proyectos competitivos entre comunidades ha sido diagnosticada también como una limitación que fragmenta y quita potencial al proceso participativo (Consejo Nacional para la Participación Ciudadana 2017) ${ }^{7}$.

En cuanto a asociatividad, en términos comparados, Chile muestra una gran cantidad de expresiones organizativas y un amplio repertorio de formas de acción colectiva. Sin embargo, lo distintivo del caso es la baja densidad de las vinculaciones entre las organizaciones y la falta de acceso a las decisiones de política a través de los canales establecidos, sobre todo en el ámbito local (Collier y Handlin 2010). Los estudios disponibles sobre asociatividad, instrumentos legales de participación local, experiencias innovadoras y percepción ciudadana, tienden a coincidir en algunos puntos comunes (Delamaza y Ochsenius 2010, Foster 2010, Sociedad en Acción 2017). En primer término, señalan que en Chile existe una alta tasa de asociatividad de primer grado (de base), con mayor densidad en zonas rurales, aunque de escasa interlocución e incidencia en políticas y orientaciones de gestión pública. En segundo lugar, los escasos instrumentos participativos dispuestos por ley son poco conocidos y se utilizan de modo irregular y parcial en la gran mayoría de los municipios del país, a pesar de la consagración legal de ciertos instrumentos. Por otra parte, las expectativas de la población sobre instancias de participación han ido creciendo en el último decenio y no se corresponden con la oferta existente. Dichas expectativas incluyen aspectos de participación política, como son las instituciones de democracia directa, como se detalla más adelante ${ }^{8}$.

La debilidad asociativa se expresa en una acentuada fragmentación y atomización de las organizaciones sociales, en especial entre los sectores más pobres y marginados. No se trata de que las personas no participen

sociales que los afectan" (Fundación para la Superación de la Pobreza 2009: 227). Dicha garantía no se implementó en el país.

7 Si bien los mecanismos contemplados en la Ley 20.500 resuelven el problema de la discontinuidad, al estar institucionalizados, su carácter consultivo y su indefinición en relación al ciclo de las políticas públicas, les han impedido abordar las otras limitaciones mencionadas. Ver más adelante.

8 Esta demanda de participación se incrementó de manera significativa a partir del 18 de octubre de 2019, cuando se inició un proceso de movilización masiva inédito, que duró hasta la declaración del estado de emergencia por la pandemia del Covid-19. Este proceso llevó a los partidos políticos a acordar un itinerario de cambio constitucional que dará origen a un nuevo período político en el país. 
en asociaciones de diverso tipo, sino más bien que este tejido asociativo carece de suficiente cohesión, está formado por unidades muy pequeñas, de escaso alcance en su accionar, poco coordinadas entre sí y con otras organizaciones. Es lo que hemos llamado el archipiélago de la sociedad civil: un conjunto de pequeńas organizaciones desconectadas, dotadas de un bajo nivel de influencia, con déficit de liderazgos, así como de condiciones materiales inadecuadas para su reproducción y acción efectivas (Delamaza 2015). Un ejemplo de esto es la situación de las Juntas de Vecinos. Estas organizaciones territoriales, existentes en todas las comunas del país, amparadas legalmente desde 1968 (Ley 16.880) no cuentan con un lugar en la gestión municipal, no tienen financiamiento, ni escuelas de formación de dirigentes. Sin embargo, son las que son las organizaciones que más crecen en número, 4,7 veces entre 2006 y 2015, llegando a representar el $79,8 \%$ de las organizaciones comunitarias funcionales y uniones comunales de juntas de vecinos (Centro de Políticas Públicas 2016). Al no poder unificarse por territorio, ni contar con espacio de conexión con la gestión municipal, esta multiplicación se traduce en fragmentación y falta de incidencia, aspecto en el cual coinciden todos los actores (Biblioteca del Congreso Nacional 2018)

También en este plano las diferencias sociales se expresan en desmedro de los sectores más pobres y marginados: los sectores socioeconómicos altos cuentan con mayor dotación de capital social tanto formal como informal (Programa de Naciones Unidas para el Desarrollo 2000). Y al no estar garantizada la participación como un derecho, incluso en el ámbito de los movimientos sociales y la acción colectiva contenciosa, la desigualdad se reproduce. Así, un estudio realizado en Santiago, muestra como los movimientos ciudadanos urbanos de sectores pudientes, se desarrollan más rápido y obtienen mayor satisfacción a sus demandas que los de los grupos excluidos (Poduje 2007).

Pero más allá de las organizaciones, el fenómeno de la falta de incidencia de la participación se expresa en el plano subjetivo como una falta de reconocimiento, e incluso una percepción de "invisibilidad" de los sujetos frente al Estado, a lo que se suma la percepción de impotencia, vale decir la escasa capacidad de producir cambios a partir de la propia acción (Fundación para la Superación de la Pobreza 2013). Este fenómeno no se limita a una pequeña fracción de la población, sino que se registra como un algo prevalente en un porcentaje cercano al tercio del total, aquella que 
intermitente o establemente se ha encontrado en situaciones de pobreza de ingresos?.

A partir de 2005 y con mayor intensidad y amplitud desde 2011-2012, se evidencia en el país una creciente demanda social por una participación efectiva. La democracia chilena se encuentra atravesando por múltiples problemas que van desde crecientes cuestionamientos a la élite política hasta insatisfacción con los espacios y mecanismos de participación ciudadana. Los avances contemplados en la Ley 20.500, poco conocidos, han sido cuestionados por parte de la sociedad civil organizada, que demanda una participación ciudadana efectiva, es decir, que implique consecuencias visibles y mayor utilidad (Garretón 2017). Por ejemplo, en 2016, durante el segundo gobierno de Michelle Bachelet (2014-2018), se dio inicio a una etapa de participación ciudadana para un proceso constituyente que luego se plasmaría institucionalmente. La demanda por plebiscitos, referendos y consultas ocupó el primer lugar de las preferencias ciudadanas por nuevas instituciones para la Constitución Política en cada una de las diferentes modalidades del proceso (consultas individuales, encuentros locales autoconvocados y cabildos provinciales y regionales). Allí "se propone fundamentalmente la necesidad de que haya participación ciudadana directa y vinculante en las decisiones. Esta participación debe materializarse en mecanismos como los plebiscitos, consultas y referendos" (Comité de Sistematización 2017: 33).

Pero la expresión más importante de dicha demanda de participación ha sido, hasta ahora, el conflicto y la movilización social, sin canales institucionales para sus reivindicaciones. Así sucedió con el movimiento por la educación pública (a partir de 2011) y así ha sucedido también con los diversos conflictos socio-territoriales en torno a problemas con la industria extractiva, la producción energética, la disponibilidad y acceso al agua en sectores rurales, la contaminación, la dotación de servicios públicos de salud en diversas ciudades intermedias del país, la demanda

9 El fenómeno tiene consecuencias políticas negativas para la democracia, pues incrementa el distanciamiento de la población respecto de la representación política institucional, fenómeno agudo sobre todo entre los jóvenes y, entre ellos, los jóvenes populares. La desafección y desconfianza institucionales son muy altas en el país (Programa de Naciones Unidas para el Desarrollo 2014) y se ampliaron a la mayoría de las instituciones tanto económicas como políticas, con la explosión pública de escándalos de corrupción política, colusión empresarial y otros a partir de 2014. El levantamiento social de fines de 2019 mostró con dureza las consecuencias de este distanciamiento, pues no reconoció conducción de ningún actor político y se expresó en ataques a diversas instituciones económicas y políticas, el sistema de transporte público, símbolos urbanos, etc. 
por restitución de tierras indígenas, entre otros (a partir de 2005, con un peak en 2012) (Delamaza 2017). Esta reactivación social amplia contrasta con la escasa respuesta institucional que permita dar cauce a esas demandas a través de mecanismos de participación adecuados.

En síntesis, durante un tiempo prolongado, la oferta pública de espacios legítimos, mecanismos efectivos y derechos de participación para la ciudadanía en los asuntos públicos fue escasa. Y lo poco que hubo, fue de escasa calidad: funcional para los responsables estatales, pero casi nada para las y los ciudadanos en términos de incremento de su incidencia. Cuando no directamente inocua en sus resultados y poco fiable en sus promesas. Durante un tiempo ello coincidió con una demanda también difusa por participar. En especial los años noventa no destacaron en cuanto a una ciudadanía empoderada y demandante. Como sabemos, ello comenzó a cambiar rápidamente a mediados del decenio pasado y dramáticamente desde la coyuntura 2011-2012: activación de nuevos sectores, temas más amplios y profundos y una movilización mucho más explícita fueron los signos de ese proceso. El movimiento por la educación pública, los innumerables conflictos socio-territoriales y la masividad del movimiento en contra de las Administradoras de Fondos de Pensiones (AFP) (20162017), son claros indicadores de ello. También lo es la amplia respuesta obtenida en la etapa participativa del proceso constituyente. A pesar del brevísimo tiempo del que se dispuso, la escasa difusión que este tuvo y lo abstracto de los temas que se abordaban, la participación alcanzó a más de 200.000 (OCDE 2017). El proceso experimentaría un nuevo salto luego del levantamiento social de octubre 2019. En diciembre de 2019, la Asociación Chilena de Municipalidades (ACHM) organizó en Chile una consulta sobre la necesidad de contar con una nueva constitución por medio de una Asamblea Constituyente. Participaron en ella casi 2.500.000 de personas, con una aprobación abrumadora a ambas alternativas (El Mostrador 2019) ${ }^{10}$.

En consecuencia, la sociedad se ha estructurado principalmente sobre la base de la competencia y la iniciativa individual. Sus estructuras institucionales son percibidas como incapaces de procesar los conflictos y proporcionar los bienes públicos que prometen (seguridad y protección, ciudadanía igualitaria, educación y movilidad social, participación pública, entre otros) (Garretón 2017, Ruiz 2017) La continuidad del crecimiento económico durante la etapa del boom de los commodities produjo dos fenómenos que, lejos de disminuir el problema, lo agudizaron: de una

10 De esa consulta se restaron las municipalidades de las comunas más acomodadas de Santiago. 
parte, difundió una imagen de éxito del país que pone en evidencia aún más la inequidad existente para disfrutar de los beneficios de dicho éxito. De otra, incrementó las expectativas, tanto de quienes experimentaron cierta movilidad social y quisieran continuarla y consolidarla, como de quienes observan que las reglas del juego resultan injustas respecto de importantes grupos en la sociedad (Peña 2020).

El escenario de alta demanda y baja respuesta, anticipa frustración y conflicto. En cambio, uno en que la oferta política se haga cargo de los aspectos fundamentales de la demanda ciudadana permite pensar en un avance democratizador significativo y la restauración de lazos entre ciudadanía y política, que se encuentran en un muy mal momento. La demanda de participación lejos de ser una amenaza, como el establishment político tendió a verla durante mucho tiempo -y en muchos casos sigue haciéndolo-, es una inmensa oportunidad para la democracia. Es una respuesta efectiva a la desafección, la rabia, la tentación populista o autoritaria y otros fenómenos hoy en boga en Chile y el mundo.

\section{PARTICIPACIÓN CIUDADANA Y POLÍTICAS PÚBLICAS PARA SUPERAR LA POBREZA: UN ENFOQUE CONCEPTUAL}

Una forma de entender la pobreza es como el reflejo o consecuencia de fenómenos mayores (distribución del poder). Los sujetos serían más pobres en la medida que no logran una suficiente dotación de poder propio que les permita participar e influir en las decisiones que les atañen. Es lo que refleja el viejo aforismo "más tiene el rico cuando empobrece que el pobre cuando enriquece". En la medida que los ingresos y recursos económicos son la consecuencia del acceso a los mecanismos que los producen -las redes, los lugares, la información, las oportunidades, el capital social y cultural de las familias- la participación en decidir cómo se estructuran esos mecanismos es un factor sustantivo de la pobreza, sus causas y evolución.

Al mismo tiempo, también se puede considerar que la participación representa una necesidad consustancial a la existencia humana. Es decir, los seres humanos no podemos abstraernos del acto de participar y tomar parte interactivamente con nuestro medio. No hacerlo o hacerlo a través de satisfactores inadecuados, podría evidenciar una situación de pobreza o engendrar ciertas patologías sociales. (Fundación para la Superación de la Pobreza 2009) Los enfoques del desarrollo humano ponen en el énfasis no solo en la obtención de bienes materiales y su distribución (crecimiento y equidad), sino también en un aspecto clave que involucra a los sujetos: desarrollo es aquello que el sujeto -individual y colectivamente- define como tal y no algo definido externamente por algún experto o agente 
modernizador. Superar la pobreza y obtener mayor bienestar supone, entonces, definir previamente ese bienestar deseado y las acciones legítimas para lograrlo. En términos discursivos la superación de la pobreza aparece crecientemente vinculada a estos enfoques de desarrollo humano (Conconi y Viollaz 2018). Si se compara la evolución de las políticas sociales en América Latina desde las reformas neoliberales de los ańos ochenta y noventa, respecto de la realidad durante el "giro a la izquierda" (20072014 aproximadamente) e, incluso, bajo gobiernos de derecha, también se aprecia un acercamiento de dichas perspectivas. Sin embargo, la convergencia es relativamente lenta, puesto que los índices de pobreza se siguen midiendo en términos de "sacar de la pobreza" a cierta cantidad de personas. Aspectos como la participación normalmente no están integrados entre los indicadores que los estados utilizan ${ }^{11}$.

En términos muy prácticos, el vínculo que proponemos entre participación y superación de la pobreza se desprende del núcleo mismo del enfoque de desarrollo humano (PNUD 2015). En este se asume que la participación efectiva en los asuntos que a cada uno conciernen incrementa las posibilidades de que las decisiones incluyan los puntos de vista e intereses de quienes tuvieron la oportunidad de expresarlos y hacerlos valer, tendiendo a desfavorecer los de quienes no accedieron a participar. Es lo que ocurre con frecuencia a los grupos en situación de pobreza: si no participan, sus problemas, intereses y propuestas muchas veces no alcanzan siquiera a hacerse visibles. Al contrario, los intereses dominantes en una sociedad se hacen presentes por múltiples vías ante los tomadores de decisiones, incluso sin necesidad de participar de instancias formales (muchas veces prefieren no hacerlo). Cuentan con las capacidades de participar y las ejercen de diversa forma: formulan propuestas, las difunden ampliamente, construyen argumentos para legitimarlas $y$ defenderlas, se comunican directamente con quienes toman decisiones, las institucionalizan en instancias formativas y de comunicación masiva, ejercen presión, etc. Por lo tanto, se requieren acciones afirmativas para ampliar la participación a quienes nunca participan por razones de

11 En el caso chileno, recién en 2015 se modificó la forma de medición de la pobreza, incorporando un enfoque multidimensional de la misma, basado en el desarrollado por Alkire y Foster (2007). Dicho enfoque, sin embargo, no incluye ni acción colectiva, ni participación institucionalizada, ni evaluación de las personas sobre su propia situación. La Encuesta de Caracterización Socio-Económica Nacional (CASEN) incluyó durante dos versiones una pregunta sobre participación en organizaciones, la cual fue omitida con posterioridad. Ver por ejemplo la versión 2017 de la Encuesta CASEN en http://observatorio.ministeriodesarrollosocial.gob. cl/casen-multidimensional/casen/casen_2017.php [03-03-2020]. 
discriminación, lejanía, falta de recursos, etc. Y, al mismo tiempo, crear condiciones de una deliberación efectiva y equilibrada.

Desde nuestro punto de vista, todos los programas, decisiones y acciones públicas son participativas. Vale decir, que la participación siempre está presente en cada uno de esos fenómenos. No son procesos automáticos, ejecutados sin actores que los promuevan, organicen e implementen. Tampoco son procesos que dependan exclusivamente de una autoridad individual -como pueden serlo en una dictadura personalista- sino que comprometen a diversas personas en distintas etapas. En la sociedad moderna, por otra parte, los asuntos públicos han visto incrementada la complejidad de los problemas que abordan. La propia definición de lo que es público y debe ser abordado por las políticas públicas se ha vuelto materia de disputa, no solo por razones ideológicas, sin por la pluralidad de voces y actores que en ello intervienen ${ }^{12}$. Ello ha incorporado también a más actores en los procesos de toma de decisiones e implementación de acciones. Ya no solo basta con la legitimidad de la autoridad legal (los que mandan) o la proveniente del conocimiento experto (los que saben). Las competencias legales se distribuyen y amplían a través de procesos de transferencia de atribuciones a distintos niveles y algo similar ocurre con los diferentes tipos de conocimiento y saberes en sociedades complejas.

De tal manera que el problema de la participación no es de si ella existe o no existe: siempre existe. Si ello es así, las preguntas relevantes para efectos de relacionar estos procesos con los de superación de la pobreza son otras. En primer término, ¿quiénes participan y quienes quedan excluidos de estos procesos? Nunca un mecanismo participativo es inclusivo por completo, ni homogéneo en cuanto a su acceso o ejercicio, puesto que existen barreras de entrada, disponibilidad de información y otros factores que van permitiendo o fomentando su mayor o menor utilización y apropiación por parte de diferentes grupos sociales. Aquí no interesa especialmente la cantidad de participantes sino, en particular, si está incorporada o no la diversidad de actores implicados en una política o programa, así como su inclusividad respecto de quienes tienen menores oportunidades para participar.

12 ¿Deben garantizarse legalmente los derechos sociales? ¿Debe priorizarse el crecimiento económico o el desarrollo sustentable? ¿Hasta dónde y cómo debe intervenir el Estado en asuntos que competen a decisiones de los individuos? ¿Cómo se resguardan los derechos y demandas de autonomía de las comunidades indígenas en el marco de un Estado nacional? Estas y muchas otras son preguntas que no tienen una respuesta única que pueda ser considerada legítima para y por todos. 
Pueden citarse dos ejemplos chilenos que indican diferentes situaciones en esta materia. En el caso de los plebiscitos comunales de origen ciudadano, legales desde 1999, solo la comuna de Vitacura -la comuna más rica de Chile- ha podido realizar uno en los veinte años de vigencia de la ley que los regula, por las importantes barreras de entrada, entre ellas el costo, que implica su realización (Programa de Naciones Unidas para el Desarrollo 2014). Vale decir que, tal como está regulado, solo la comuna de mayores ingresos económicos ha podido utilizarla. Un ejemplo en contrario es la llamada Ley Lafkenche, (Ley de Espacios del Borde Costero para Pueblos Originarios, de 2007), que permite reconocer derechos sobre el borde costero a comunidades indígenas que así lo soliciten y que demuestren su uso consuetudinario del mismo, dándoles prioridad por sobre otras solicitudes (Delamaza y Flores 2012). De modo tal que la pregunta ¿participan los pobres?, tiene dos respuestas contrapuestas en estos ejemplos: mientras los plebiscitos comunales tal como están diseñados los excluyen, la Ley Lafkenche ha permitido la incorporación de un actor anteriormente excluido a un bien como el borde costero ${ }^{13}$.

Un segundo aspecto a considerar es cómo se participa. Vale decir, los mecanismos concretos que se utilizan: los lenguajes, los horarios, los lugares, la distribución de los costos, los métodos de trabajo, la construcción y transparencia de los liderazgos, el potencial deliberativo y el alcance del mecanismo son importantes de considerar. Ninguna de estas características es neutra, especialmente en sociedades donde los recursos de todo orden están desigualmente repartidos. Un ejemplo simple es el financiamiento del transporte para llegar al lugar donde se realizan reuniones u otro tipo de actividades de participación: para una persona de mayores recursos se trata de un costo marginal, que no incide en su disposición a participar; para una persona de escasos recursos se trata de una decisión relevante ${ }^{14}$.

13 Pocas instituciones evalúan esta dimensión clave de la participación. Una de las que sí lo hace es el Consejo para la Transparencia, que elabora anualmente un Informe donde establece cuantos conocen el derecho al acceso a la información pública, quienes están dispuestos a ejercerlo y cuál es la proveniencia social de quienes hacen uso del mismo. Los resultados de estas evaluaciones indican que el conocimiento de la instancia donde reclamar, la disposición donde hacerlo y el ejercicio efectivo de las reclamaciones ante el consejo por denegación del derecho son bajas y no crecen a lo largo del tiempo. Al mismo tiempo son directamente proporcionales al nivel socioeconómico de las personas (Consejo para la Transparencia 2016). El ejercicio del derecho a reclamo frente a la denegación tiende a concentrarse en sectores urbanos y de alta educación (Díaz-Tendero 2014)

14 Aplicado al ámbito electoral, es por ello que se considera que la carencia de transporte público gratuito durante los días de elecciones es un factor a favor de la elitización del 
También la disponibilidad de información pertinente, comprensible y oportuna es un factor clave. Un caso que indica los problemas en tal sentido es el de la participación en los proyectos sometidos al Servicio de Evaluación de Impacto Ambiental (SEIA), mecanismo establecido para todos los proyectos productivos o de infraestructura que tienen diversos impactos en el medio ambiente. Mientras los titulares de los proyectos -los inversores- cuentan con todo tipo de recursos técnicos y legales para la formulación de sus proyectos antes de presentarlo al SEIA, las comunidades locales disponen de un período restringido (60 días) para procesar asuntos de gran complejidad técnica, sin contar para ello con ningún tipo de asistencia técnica, más allá del que puedan por sí mismas gestionar. En comunidades pobres, por cierto, esos son recursos escasos. Combinando las dos preguntas tendríamos la siguiente: ¿Quiénes tienen acceso a qué tipo de mecanismos?

La tercera característica a resaltar en los mecanismos y formas de participación son las consecuencias del proceso participativo, que responde a la pregunta simple de para qué participar. En cuanto a los objetivos perseguidos, podemos distinguir entre una participación sin mayores consecuencias o decorativa; una de tipo instrumental o funcional a las metas de la política pública y aquella de tipo empoderadora, que fortalece las capacidades de la comunidad o los grupos sociales. Esta última dimensión es fundamental cuando se trata de grupos marginados o excluidos. Por una parte, tienen mayores dificultades, organizativas y de otro tipo, para sumarse a la tarea de la participación. Pero, por otra, ven en la participación una herramienta de fortalecimiento. El predominio del enfoque principalmente instrumental, como ocurre en Chile, es un elemento desfavorable para la participación de los grupos pobres.

Una segunda dimensión en relación a las consecuencias de participar es, sin duda, el alcance de la misma, vale decir si refiere a la definición de los temas a abordar (formación de la agenda), a la toma de decisiones, a la formulación de programas y proyectos concretos, a la implementación de acciones y/o al control social y retroalimentación transformadora de la política. También es importante si la participación es concebida como un derecho adquirido que debe ser garantizado y exigible o si se trata solo de una acción que la autoridad decide emprender por cualquier motivo. Finalmente, adoptamos un indicador conceptualizado por Cunill (2004) como "recursos directos de poder institucionalizado", que pueden ejercer las instancias de participación sobre la administración pública. La autora distingue entre poder de certificación, asignación y veto. El desempeño

voto en Chile. 
de cada una de estas dimensiones -objetivos, alcance, derecho y recursos de poder- definirá las consecuencias concretas que la participación puede tener tanto sobre los participantes mismos como sobre la dinámica y evolución de las políticas.

Un último aspecto a considerar se refiere al debate sobre la efectividad de la participación en la superación de la pobreza, más allá del valor intrínseco que se le conceda al acto de participar. Vale decir, ¿son más costo-efectivos los programas que incorporan un enfoque de participación más fuerte? Como se comprende, la pregunta es difícil de responder en general, precisamente por la diferencia de contenidos concretos que tienen cada una de las modalidades y enfoques de la participación ${ }^{15}$. En el caso chileno, además, existe muy poca evidencia empírica al respecto: no hay centros de investigación dedicados al tema, el Estado no evalúa sus acciones participativas, limitándose a la estadística de su puesta en práctica ${ }^{16}$. Sin embargo, muchas veces se utiliza como argumento para señalar la dificultad de implementar procesos participativos la falta de recursos -asumiendo que esta encarece los programas y acciones- su mayor "lentitud" - pues consultar supondría demorar- y la poca utilidad de consultar opiniones de por sí diversas para tomar decisiones que requerirían de fundamentos técnicos. En cuanto al mayor costo, al menos en la experiencia chilena, tanto las metodologías participativas utilizadas por el Ministerio de Obras Públicas como el reciente enfoque de participación y diálogo del Ministerio de Energía sugieren exactamente lo contrario: fueron puestas en marcha a raíz de la conflictividad en torno a las inversiones y su consecuente costo de judicialización, demora, indemnizaciones. O directamente la imposibilidad de realizar ciertas inversiones ${ }^{17}$. En relación al mayor tiempo

15 Por ejemplo, Montecinos (2007) analiza las semejanzas y diferencias existentes entre la participación considerada en los Planes de Desarrollo Comunales (Pladeco) y los presupuestos participativos.

16 Desde hace poco tiempo, el Sistema Nacional de Información Municipal (SINIM) reporta la existencia de Ordenanzas Municipales de Participación y de COSOC en los municipios (www.sinim.gov.cl). En el caso de los otros mecanismos de orden sectorial, la División de Organizaciones Sociales del gobierno ha hecho el seguimiento estadístico, pero no ha difundido información fiable y completa.

17 Sobre el Ministerio de Obras Públicas, ver: http://especiales.mop.gov.cl/participacion/ wp-content/uploads/2010/08/Manual_PAC.pdf Sobre la División de Participación y Diálogo del Ministerio de Energía, ver http://www.energia.gob.cl/que-entendemospor-participacion-ciudadana Un análisis de las consecuencias de los conflictos socio territoriales sobre las decisiones de política pública en Delamaza, Maillet y Martínez (2017). 
requerido, nos parece que el problema principal es más bien la diferencia que puede existir entre el "tiempo administrativo" de las políticas públicas, sometidas tanto a la burocracia y el control legal y administrativo, como a la presión "gerencialista" del enfoque predominante en las estrategias de modernización del Estado inspiradas en la Nueva Gestión Pública (Cejudo 2010), que no han internalizado estas variables en su diseńo y procedimientos. Finalmente, en lo relativo al predominio del saber técnico, se trata de una materia altamente controvertida, puesto que la complejidad social avala un enfoque de "diálogo de saberes" y la necesidad de legitimidad social de las políticas.

La vinculación entre participación y superación de la pobreza es múltiple. En sí misma, la falta o imposibilidad de participar en asuntos que me atañen directamente es ya una forma de pobreza, puesto que las decisiones me son impuesta por otros, que sí participan (siempre alguien lo hace). Pero, además, lo más probable es que aquellas decisiones en las que tengo participación me beneficien o tomen en cuenta de manera más clara y adecuada, mientras que, si no tengo esa opción, los resultados seguramente beneficiarán más a otros. El fortalecimiento de una política pública participativa pasa por el reconocimiento del derecho humano a participar de todas las personas y comunidades. Ello significa, en contextos de gran desigualdad, que no basta con abrir oportunidades de hacerlo, estableciendo "mecanismos participativos". Sobre todo, si se quiere vincular el objetivo de participación con el de superación de la pobreza.

Además de la existencia de mecanismos, un enfoque de participación efectivo exigiría:

- Considerar mecanismos que "emparejen la cancha" de la participación hacia los grupos marginados, excluidos y/o discriminados, reduciendo las asimetrías de poder y creando condiciones adecuadas para la deliberación, el diálogo y la resolución conjunta de los asuntos concernidos.

- Incorporar la participación como una política transversal, respaldada por recursos públicos, evaluable en sus logros y dificultades en un marco de desarrollo estratégico; puesto que es difícil que este avance de manera sostenida si no forma parte de los objetivos mismos de la política pública, más allá de su dimensión instrumental.

- Una importante inversión metodológica, de capacitación de actores (institucionales y sociales) y adecuación de incentivos, que acompañe la voluntad política y permita una adecuada implementación ya que no se trata solamente de establecer medidas administrativas. 
El desafío es modificar las prácticas (asistenciales, de dependencia, asimetría y subordinación) y eso supone desarrollo de capacidades específicas para lograrlo.

\section{¿QUÉ SE HA HECHO, HASTA AHORA, EN MATERIA DE PARTICIPACIÓN Y POBREZA?}

En concordancia con el enfoque predominante sobre participación, que privilegiaba lo electoral, desde un inicio se buscó restablecer los procedimientos de elección para las diferentes ramas del poder (Ejecutivo y Legislativo con severas restricciones hasta 2005) y niveles (municipios, a partir de 1992, y regional, parcial e indirecto desde 1993). Pero la participación autónoma de los movimientos sociales comenzó a ser vista más como una amenaza a la gobernabilidad democrática que como una contribución a ella. Y en el campo institucional se modificó solo parcialmente la estructura local establecida por la dictadura (los Consejos de Desarrollo Comunal, CODECO) a través de los Consejos Económico Sociales Comunales (CESCO), cuya función fue más que nada decorativa, sin lograr adhesión por parte de la población, ni tampoco de parte de los alcaldes o de sus propios integrantes. Salvo el SEIA, a través de la Ley de Bases del Medio Ambiente de 1994, no se institucionalizó tampoco otra modalidad de participación en asuntos públicos.

La vía utilizada para la participación social fueron los mecanismos ad hoc diseñados por algunos programas gubernamentales de aplicación local, principalmente orientados a la extrema pobreza. El más continuo de ellos ha sido el de pavimentos participativos (luego pavimentación participativa) mediante el cual los ciudadanos de comunidades pobres cofinancian la pavimentación de sus calles y pasajes, subsidiando así al Estado, que no cuenta con suficientes recursos. Allí, participación equivale a cofinanciamiento y se acota a la realización de proyectos muy específicos. $\mathrm{Ni}$ el recién mencionado, ni otros programas ejecutados a través de pequeños proyectos competitivos, incluyeron objetivos de participación en decisiones $\mathrm{u}$ orientaciones de la política y los programas. Tampoco se institucionalizaron como un derecho ciudadano, dependiendo su continuidad de la voluntad de la autoridad.

Una innovación interesante se produjo en el ámbito de las políticas de vivienda con la introducción del programa Quiero mi Barrio, a partir de 2006. En él se incluyó una planificación participativa local a través de un consejo local que se coordina con el ministerio, pero sin la suficiente participación ni articulación con el municipio, ni tampoco con una 
institucionalización en el territorio, a través de las Juntas de Vecinos u otra instancia permanente.

En 1999 se modificó la Ley Orgánica Municipal, introduciendo en ella tres mecanismos de participación: la audiencia pública, las ordenanzas municipales de participación ciudadana y los plebiscitos comunales. Mediante la primera, un número determinado de vecinos puede solicitar la presencia del Concejo Municipal para tratar un problema específico (se influye así en la agenda municipal). Las ordenanzas norman la participación de manera diferente en cada comuna. Los plebiscitos pueden ser convocados por el Alcalde o por los ciudadanos, previa reunión de un número de firmas, en torno a materias muy precisas de inversión municipal. Los resultados de dichas modificaciones son indicativos del diagnóstico que hemos propuesto. Al ser materias reguladas por ley, los mecanismos fueron institucionalizados, de acuerdo a la máxima que tanto se repite en Chile: las instituciones funcionan ${ }^{18}$. Pero, en términos de operatividad y efectividad, los resultados han sido magros, puesto que las ordenanzas fueron mayoritariamente establecidas sin deliberación ciudadana y siguiendo un modelo estándar propuesto por la Asociación Chilena de Municipalidades, sin flexibilización y apropiación local. Y en el caso de los plebiscitos, estos tienen un alto costo y barreras de entrada difíciles de superar, lo cual ha significado que en más de una década y media apenas se han realizado cuatro de ellos, en comunas de altos ingresos, de los cuales solo uno de origen ciudadano (Vitacura) (Programa de Naciones Unidas para el Desarrollo 2014, Consejo Nacional de Participación Ciudadana 2017).

En el ámbito sectorial, desde mediados de los noventa se estableció por ley el SEIA, utilizado para algunos de los proyectos con externalidades ambientales que estaban incrementándose en el país. Medio ambiente fue el único sector que institucionalizó un sistema, aunque con enormes limitaciones o, más precisamente, con enormes asimetrías entre los actores concernidos: de una parte, grandes empresas nacionales o internacionales, protegidas legalmente y dotadas de los recursos técnicos, comunicacionales y políticos; del otro, comunidades rurales y urbanas, muchas veces aisladas y con escasos recursos, carentes de cualquier apoyo técnico, que entraban en el proceso una vez presentado el proyecto, generalmente de gran complejidad técnica, económica y jurídica. A partir de ese momento, los grupos directamente afectados disponían de 60 días para realizar

18 En 2009, la Fundación para la Superación de la Pobreza indicaba que con respecto a la Ordenanza de Participación "el 79\% de los municipios ya cuenta con esta herramienta” (Fundación para la Superación de la Pobreza 2009: 233). 
observaciones a los aspectos ambientales del proyecto. El SEIA es un buen ejemplo del modo predominante de entender la participación: las observaciones no necesariamente debían ser tomadas en cuenta; las empresas contaban con un mecanismo no sometido a participación (las adenda); el plazo y las condiciones de la participación no permitían practicarla seriamente $\mathrm{y}$, por último, todo el proceso comenzaba recién cuando los proyectos estaban completamente formulados y localizados, perdiéndose la valiosa oportunidad de deliberar entre empresas y comunidades sobre el emplazamiento, características y destino de los proyectos. No es de extrañar, en este contexto, que la conflictividad en torno a proyectos con impactos ambientales negativos se haya incrementado significativamente a partir de 2005 en adelante (Instituto Nacional de Derechos Humanos 2015, Consejo Nacional de Innovación para el Desarrollo 2016), sin encontrar cauces adecuados a través de los mecanismos institucionales.

Los mecanismos legales que regularon la participación desde 1990 y hasta 2007, aproximadamente, tuvieron varios rasgos comunes: se trató de mecanismos a escala local, escasos en cantidad y variedad y que cubrían solo unas pocas áreas de acción municipal o sectorial (medio ambiente); fueron de participación directa (consultas) y a través de representantes (consejos), pero poco masiva en su práctica y en su mayoría excluyente de los sectores pobres y marginados (plebiscitos). La participación se entiende en ellos como una práctica positiva de alcances muy limitados (entre decorativa e instrumental). Esta descripción muestra que la participación no fue una prioridad, por lo que sus efectos fueron también poco significativos tanto en la reorientación de las políticas públicas, como en incrementar la adhesión de la ciudadanía a dichas políticas (Delamaza 2012).

A comienzos del gobierno de Ricardo Lagos (2000-2006) se inició la discusión de una ley de participación, que garantizara el derecho a la misma en la gestión de los asuntos públicos. Era una forma de reaccionar a la crisis política del conglomerado en el poder -que había perdido gran cantidad de votantes- a través de medidas de modernización del Estado que no alteraran la estructura política existente. Este proceso ha durado hasta la actualidad y ha dado lugar a que en cada período de gobierno se dicte un nuevo Instructivo Presidencial de Participación Ciudadana, destinado a la administración ejecutiva; a la creación de un pequeño Fondo de Fortalecimiento de Organizaciones de la Sociedad Civil en 2003 (posteriormente llamado Fondo de Fortalecimiento de Organizaciones de Interés Público (FOIP) e incluido en la Ley 20.500) y a la discusión legislativa del proyecto de Ley de Asociatividad y Participación Ciudadana en la Gestión Pública que llevaría a su promulgación en febrero de 2011 (reglamentada y comenzada a aplicar en febrero de 2012). 
La ley establece que los órganos del Poder Ejecutivo deben dictar normas en la materia y constituir consejos de carácter consultivo. En el plano municipal sustituye los antiguos CESCO por los COSOC, también consultivos. Asimismo, establece ciertas obligaciones nuevas para alcaldes, Concejo Municipal y Secretario Municipal. Finalmente, facilita la constitución de organizaciones y entrega algunos recursos de financiamiento para las dichas organizaciones a través del FOIP. El Informe solicitado por la presidenta Bachelet estableció varios puntos críticos que debilitan la Ley 20.500. Entre los principales se citan la falta de financiamiento a los mecanismos establecidos y la falta de sanción al incumplimiento de la norma por parte de las autoridades. También se plantea la falta de articulación entre las instancias participativas y el ciclo de las políticas públicas. Otro aspecto relevado es la inexistencia de mecanismos participativos vinculantes a decisiones y la reducción de la participación a la "consulta". Finalmente, se considera que los recursos para el fortalecimiento de la asociatividad son el punto más débil de la ley, debiendo fortalecerse (Consejo Nacional de Participación Ciudadana 2017).

A partir de mediados de los ańos 2000 se promulgaron diversas iniciativas legales que se relacionan con este tema: 1) la Ley 20.285 de Transparencia de la Función Pública y de Acceso a la Información de la Administración Pública (2007) que amplía significativamente los derechos a información pública de los ciudadanos; 2) la ratificación del Convenio 169 de la OIT sobre Pueblos Indígenas y Tribales (2009) que garantiza y regula la consulta previa, libre e informada sobre proyectos públicos que involucren a los pueblos indígenas; 3) la Ley 20.609, que establece medidas contra la discriminación, conocida como Ley Zamudio (2012); 4) la modificación de la Ley de Bases del Medio Ambiente (2007) que amplía los proyectos sometidos a participación ciudadana.

En materia de programas sociales, a partir de mediados de los años 2000 se introdujo la noción de "derechos garantizados" en la gestión pública del área social. Sin embargo, llama la atención que los programas que involucran la garantía de derechos ciudadanos no fueron acompañados de instancias participativas de la comunidad, ni en su diseño ni en su implementación y control, como ocurrió en los casos del Sistema Chile Solidario (hoy Sistema de Protección Social) y la reforma de salud ${ }^{19}$. El

19 El Sistema Chile Solidario establece un conjunto de prestaciones estatales garantizadas para la población de bajos ingresos. Con posterioridad se incluyó un capítulo especial para las niñas y niños (Chile Crece Contigo) y los adultos/as mayores, creándose el Sistema de Protección Social (Robles 2013). En la Reforma de Salud se optó por un 
enfoque familiar del primero y sus derivados y el enfoque centrado en la atención individual en salud, no incluyen una orientación participativa, más allá de la de quejas y reclamos. Por su parte tampoco la política de participación ciudadana en la gestión pública (que culminó en la Ley 20.500) estuvo vinculada a las metas y prioridades de la política social (educación, salud, pobreza). Así, creemos que no ha existido una sinergia y acoplamiento entre la agenda modernizadora del Estado y su componente de participación ciudadana con respecto a la política social y sus programas más o menos participativos. Como tampoco ello ocurrió con la agenda emergente de garantía de derechos universales, ello convierte a la participación en un ejercicio de bajo impacto e interés para las personas ${ }^{20}$.

En los cuerpos legales de los últimos diez años la participación aparece como un derecho a ser garantizado de manera explícita. La Ley de Transparencia, la Ley Lafkenche y el Convenio 169 introducen mecanismos más fuertes de participación. No ocurre lo mismo con la Ley de Participación y la de Medio Ambiente que, aunque reconocen el derecho a participar, permanecen centradas en modalidades consultivas, sin mayores consecuencias jurídicas, de seguimiento o de sanción en caso de incumplimiento. En el caso de la Ley 20.500 tampoco contempla mecanismos de financiamiento para las nuevas tareas de participación encomendadas a los servicios públicos y a los municipios. Tampoco se consideraron las asimetrías que deben compensarse en el caso de grupos excluidos para volverla efectiva (información, capacitación, soporte técnico, financiamiento) ${ }^{21}$. En cambio, la Ley Lafkenche, que busca

Sistema de Garantías Explícitas en Salud que ha ido incorporando las 85 patologías más prevalentes en la población como "garantizadas". Ver http://www.supersalud. gob.cl/difusion/665/w3-propertyvalue-1962.html [10-03-2020].

20 Adicionalmente, el Sistema de Protección Social implicó, como consecuencia no anticipada, el debilitamiento o desaparición de las instancias de organización comunitaria que apuntaban a generar medios de vida. Como no existen parámetros para evaluar las consecuencias de las medidas de política sobre la asociatividad y la participación ciudadana, estos impactos no quedan registrados como una externalidad negativa de las políticas públicas (Fundación para la Superación de la Pobreza, comunicación personal).

21 En el caso de la Ley de Transparencia, ello se traduce en el escaso conocimiento público de los derechos que garantiza y el hecho que las acciones de amparo presentadas ante el Consejo para la Transparencia provienen muy mayoritariamente de sectores urbanos, de alta educación formal y varones (Díaz-Tendero 2014). Normalmente se trata de empresas, abogados y medios de comunicación. Los sectores pobres no conocen ni alcanzan a utilizar suficientemente estos mecanismos. 
el garantizar el derecho de acceso de los pueblos originarios al espacio marítimo costero cuando este ha sido ejercido de modo consuetudinario y el Convenio 169, que establece el derecho a la consulta libre, previa e informada, se orientan hacia un sector excluido. Adicionalmente, no lo hacen en términos definidos externamente (como ocurre con la definición de extrema pobreza), sino que asumen la identidad que los propios actores se dan, reconociéndola por parte del instrumento público. Los principales problemas de esta nueva legislación parecen estar en la implementación, en parte por lo relativamente reciente de su promulgación, pero también porque se trata de modificaciones innovadoras que no van acompañadas de un rediseño de las modalidades de acción del conjunto de los servicios involucrados ni de una capacitación adecuada del funcionariado. Pero también esto ocurre por la escasez de instrumentos puestos a disposición de su apropiación ciudadana (difusión, formación y soportes técnicos) como por parte del propio aparato público (funcionarios y autoridades) (Araos et al. 2020).

\section{A MODO DE CONCLUSIÓN: LOS DESAFÍOS ACTUALES}

El desafío es pasar a una nueva etapa en participación, dando lugar a una nueva generación de políticas, que creen condiciones adecuadas para la inclusión de los grupos en situación de pobreza. Este desafío puede especificarse a través de tres líneas de acción: reconocimiento del derecho y puesta en marcha de formas de participación vinculante, articulación efectiva con el ciclo de políticas públicas y fomento a las capacidades para participar, partiendo del reconocimiento de las buenas prácticas existentes, hacia grupos con mayores desventajas relativas ${ }^{22}$.

\section{RECONOCIMIENTO CONSTITUCIONAL DEL DERECHO A LA PARTICIPACIÓN Y AMPLIACIÓN HACIA FORMAS DE PARTICIPACIÓN POLÍTICA}

La modificación constitucional será posible en la medida que se acepte modificar significativamente el enfoque en materia de participación, para hacerla más vinculante, inclusiva y efectiva. Eso supone adoptar como fundamento el que la participación ciudadana en la gestión pública es un derecho humano que debe ser garantizado constitucionalmente y que la

22 En este acápite se siguen de cerca las propuestas formuladas en enero de 2017 por el Consejo Nacional para la Participación Ciudadana, formado por 24 líderes de organizaciones de la sociedad civil convocados por la Presidenta de la República para proponer reformas en la materia (Consejo Nacional de Participación Ciudadana y Fortalecimiento de la Sociedad Civil 2017). 
democracia debe tener nuevos y mejores mecanismos de participación para incrementar el rol público de la sociedad civil chilena, más allá del sistema electoral. Así, la democracia participativa debe ser un concepto orientador para reformar la Ley 20.500 e introducir otros cambios institucionales. El planteamiento anterior implica que se considera deber del Estado fomentar y garantizar el derecho a la participación ciudadana y la asociatividad y que la relación del Estado con la sociedad civil ha de hacerse considerando el principio del respeto a la autonomía de la sociedad civil.

Concretamente garantizar el derecho a la participación ciudadana en una nueva constitución implicaría, de acuerdo al planteamiento del Consejo Nacional de Participación Ciudadana, incorporar tanto la garantía constitucional e como incluir la participación en la gestión pública dentro de los principios fundamentales del Estado de Chile.

Otra línea de propuesta es una ampliación de los ámbitos e instituciones de participación a través de mecanismos de democracia directa, de acuerdo a lo que Lissidini (2015) llama "proactivos" y "reactivos", desde "arriba" y desde "abajo". Dotar de mayor alcance a los plebiscitos nacionales actualmente considerados en la legislación, para que la ciudadanía se pronuncie sobre materias de alta relevancia nacional. Igualmente ampliar la participación a través de mecanismos de democracia participativa, referidos a la generación y vigencia de las leyes y a la selección y mandato de las autoridades. En el primer caso se propone instaurar la iniciativa popular de ley, mediante la cual la ciudadanía puede proponer proyectos de ley que el Congreso debe discutir. A ello se agrega el referéndum revocatorio (abrogativo) de leyes, que permite plantear a la deliberación y votación popular la vigencia de textos legales previamente sancionados en el Congreso. En ambos casos se requiere establecer condiciones requeridas para hacer uso de estos derechos. En relación a las autoridades se plantea reforzar el llamado voto programático, que obliga a las autoridades a presentar un programa concreto que desean implementar en caso de ser elegidos. Este mecanismo, ya contemplado en la Ley de Partidos Políticos, recientemente promulgada, se debe complementar con la elaboración participativa de planes de acción concretos a nivel comunal y regional para los que resulten electos. Esto permitirá el control social de las promesas electorales, las que deben incluir el establecimiento del referéndum revocatorio de mandato en el caso que estas promesas no se cumplan en un período determinado. 
ForTALECER EL CARÁCTER VINCULANTE DE LOS MECANISMOS DE PARTICIPACIÓN Y ARTICULARLOS DE MEJOR FORMA CON EL CICLO DE POLÍTICAS

Hasta la fecha, todos los mecanismos han sido definidos como de carácter consultivo, lo que se suma al hecho que su funcionalidad y articulación con el ciclo de políticas no está bien definido. La primera característica les quita poder como instancia y la segunda los vuelve poco relevantes y poco relacionados con aquello que importa resolver. Incluso dentro del carácter consultivo hay preguntas no respondidas con claridad: ¿quiénes deben ser consultados y qué es lo que debe ser consultado? ¿Qué se debe hacer con los resultados de las consultas? En el ámbito sectorial solo se establece legalmente que se debe contar con los mecanismos (cuentas públicas, normas de participación, consejos de la sociedad civil). Al no estar respondidas las preguntas anteriores, resulta difícil y un tanto discrecional establecer la composición y modos de funcionamiento de los consejos y los alcances de la norma. En el caso de las cuentas participativas, se han establecido ciertos estándares mínimos que todos los organismos deben cumplir.

En el caso de los instrumentos del nivel local, las funciones del COSOC han sido establecidas, pero representan un grado muy menor de lo consultivo, pues se refieren a "emitir opinión", "informar al alcalde" y asuntos similares, que realmente no le brindan un lugar relevante en la gestión del municipio. Se ha definido algo mejor algunas obligaciones para el alcalde en orden a consultar obligatoriamente sobre ciertas materias. Pero indudablemente el hecho que la instancia sea también presidida por el alcalde le resta autonomía, al tiempo que carece de funciones de control ciudadano. En el ámbito regional estas instancias no existen, por lo que se podrían crear ya con una definición diferente.

Es necesario resolver respecto de la obligatoriedad de los mecanismos y las condiciones mínimas para su implementación. En otros términos, el no cumplimiento de la normativa debiese conllevar sanciones para la autoridad responsable y, al mismo tiempo, el financiamiento de las instancias participativas debiese formar parte de los presupuestos sectoriales o locales. Ninguna de las dos cosas ocurre hoy.

Al mismo tiempo, se debe definir las instancias en base a criterios comunes mínimos para todas ellas (incluso uniformar el nombre como COSOC), definiendo sus funciones como consultivas, incidentes, vinculantes y deliberativas, según la materia que les corresponda tratar. No acotarlas a lo consultivo exclusivamente. Sus acuerdos debiesen ser vinculantes en 
materia de política participativa, que es su ámbito principal de acción. En otras materias se deberá evitar que el COSOC se convierta en un eventual actor de veto, pero se deberá aclarar que sucede ante las diferencias entre autoridad e instancia participativa. En cuanto a la composición de las instancias, recientemente se ha generalizado la elección de los integrantes. Ahora se requiere evitar la corporativización de los consejos y su separación del debate abierto y el vínculo con sus bases. Para ello debe intensificarse la publicidad y apertura en la definición de lo que allí se aborda, fortalecerse los vínculos con las otras instancias del servicio, gobierno regional o municipio según corresponda. Por último, estas instancias deben contar con recursos para desarrollar su labor: recursos técnicos, de comunicación $\mathrm{y}$ de funcionamiento.

Otro ámbito que se debiese abordar en cuanto al carácter vinculante y más articulado de las instancias de participación, es que estas deben ser monitoreadas, evaluadas y retroalimentadas. Así, se requiere saber cuánto se conoce de ellas, evaluar sus resultados en términos de propuestas que han sido acogidas o han modificado de algún modo la política pública, mensurar la evolución de las formas participativas en el desempeño de los agentes públicos (¿Aumentan los participantes? ¿Se amplía el alcance de la participación? ¿Aumenta o disminuye la inclusión de los grupos pobres? ¿Cómo afectan determinadas decisiones políticas a las organizaciones comunitarias y el capital social?, son algunas de las preguntas que debieran dar origen a estudios). Eso debiera conducir a estrategias de mejoramiento que permitan a la participación incrementar su relevancia.

\section{FORTALECER LA ASOCIATIVIDAD Y LAS CAPACIDADES PARA PARTICIPAR}

DE LAS PERSONAS Y GRUPOS EN SITUACIÓN DE POBREZA

Este ámbito es el menos atendido por la Ley 20.500. Por una parte, el FOIP cuenta con una ínfima dotación de recursos y sus modalidades de ejecución no siempre son favorables a las organizaciones comunitarias. Por otra, mecanismos como el Registro de Personas Jurídicas sin Fines de Lucro y el Catastro de Organizaciones de Interés Público no han sido implementados para favorecer y estimular las asociaciones populares. Contar con un buen Registro es fundamental, incluir en él al Catastro y extender la atribución de los Tribunales Electorales Regionales otras instancias (como los Juzgados de Policía Local) son todas modificaciones que pueden hacerse a la brevedad.

Pero el fortalecimiento de la asociatividad requiere de otras medidas más de fondo. En primer lugar, de reconocimiento hacia las formas de organización comunitaria y respaldo a las funciones que realizan. En el 
terreno legal se requiere una modificación de las Ley de Juntas de Vecinos que devuelva la capacidad de actuar como un ente de interlocución territorial, permitiendo la existencia de una sola Junta de Vecinos por cada Unidad Vecinal, entre otras modificaciones (Viveros 2018). En términos de recursos ello debiese traducirse no solo en una ampliación significativa del FOIP (de acuerdo a una proporción sobre el presupuesto nacional, por ejemplo). Se requiere una mucho mejor articulación de los diferentes fondos con que el Estado traspasa recursos a las organizaciones para sus fines propios, terminando con la discrecionalidad que existe en muchos de ellos, la duplicidad de objetivos, la reiteración de exigencias y requisitos hacia las organizaciones (un buen registro debiese resolver eso), una ventanilla única de información y postulación, entre otras medidas. Pero también el reconocimiento implica que la política pública debe ser también evaluada según las consecuencias que esta tiene sobre el tejido comunitario y la vida de las organizaciones. Si participar en ellas es considerado un valor, entonces las decisiones que se tomen deben incluir una consideración de este elemento, lo cual, hasta ahora, no ocurre.

El fortalecimiento asociativo no es solo cuestión de recursos de financiamiento, sino también de apoyo a las capacidades y habilidades organizativas, entre ellas el liderazgo y su renovación. Este rol, que en el pasado era cumplido principalmente por los partidos políticos, las iglesias y sindicatos, hoy no cuenta con vías permanentes. Una propuesta factible es contar con escuelas regionales de liderazgo, de carácter permanente, y abiertas principalmente hacia los sectores con menores recursos.

Una última dimensión del fortalecimiento se refiere a la comunicación y presencia en la deliberación pública. Es evidente la desventaja y asimetría existente para los grupos populares en el actual sistema de medios de comunicación. Por ello las formas de comunicación de base-como radios comunitarias y canales de TV locales- resultan de gran importancia. Podría considerarse financiamiento público para el desarrollo de plataformas multimediales vinculadas al mundo social organizado. Esto tendría un impacto no solo en el sector de organizaciones formales sino también en el amplio sector que utiliza formas no tradicionales y participa a través de medios virtuales principalmente. 


\section{REFERENCIAS}

Alkire, S. y Foster, J. (2007). Recuento y medición multidimensional de la pobreza. OPHI Working Paper Series, Queen Elizabeth House, Reino Unido.

Araos, F., Catalán, E., Álvarez, R., Núñez, D., Brañas, F. y Riquelme, W. (2020). Espacios costeros marinos para pueblos originarios: Usos consuetudinarios y conservación marina. Anuário Antropológico, 2020 (I), 47-68.

Avritzer, L. (2002). Democracy and the public space in Latin America. Princeton: Princeton University Press.

Biblioteca del Congreso Nacional (2018). Juntas de vecinos en Chile. 50 años. Historia y desafíos de participación. Santiago: Biblioteca del Congreso Nacional.

Blanco, I. y R. Gomá (2002). Gobiernos locales y redes participativas. Barcelona: Ariel.

Cejudo, G. (Comp.) (2010). La nueva gestión pública. México: Siglo XXI.

Centro de Políticas Públicas (2016). Mapa de las organizaciones de la sociedad civil 2015. Santiago: Pontificia Universidad Católica de Chile.

Collier, R. y Handlin, S. (2010). Reorganizing popular politics: Participation and the new interest regime in Latin America. Filadelfia: The Pennsylvania State University Press.

Comité de Sistematización (2017). Informe Ejecutivo del Comité de Sistematización. Santiago: Comité de Sistematización del Proceso Constitucional. Disponible en http://www. sistematizacionconstitucional.cl/resultados/ [31-03-2020].

Conconi, A. y Viollaz, M. (2018). Pobreza, desigualdad y desarrollo: discusión desde el enfoque de la capacidad. En varios autores, La era de la perplejidad. Repensando el mundo que conocíamos. Barcelona: Taurus.

Consejo Nacional de Innovación para el Desarrollo (2016). Informe de evaluación de los conflictos socio-ambientales de proyectos de gran tamaño con foco en agua y energía para el periodo 1998 al 2015. Santiago: CNID. 
Consejo Nacional de Participación Ciudadana y Fortalecimiento de la Sociedad Civil (2017). Informe final. Estado de la participación ciudadana en Chile y propuestas de reforma a la Ley 20.500 sobre Asociaciones y Participación Ciudadana en la Gestión Pública. CNPC: Santiago.

Consejo para la Transparencia (2016). Estudio Nacional de Transparencia. Octava Medición. Santiago: CPLT.

Cunill, N. (2004). La democratización de la administración pública: los mitos a vencer. En Bresser Pereira, L., Cunill, N., Garnier, L. Oszlak, O. y Przeworski, A. (Eds.), Política y gestión pública. Buenos Aires: Fondo de Cultura Económica, CLAD.

Delamaza, G. (2012). Chile: la institucionalización progresiva de una participación acotada en el marco de la democracia elitista. Revista del Centro de Estudios Políticos, 7, 176-205.

. (2015). Enhancing democracy: Public policies and citizen participation in Chile. Amsterdam: CEDLA y Berghahn Books.

(2017). Sociedad civil, ciudadanía, movimiento social en el Chile de hoy. En Garretón, M. A. (Coord.), La gran ruptura. Institucionalidad politica y actores sociales en el Chile del siglo XXI. Santiago: LOM.

Delamaza, G. y Flores, F. (2012). Estrategia política territorial y reconocimiento de derechos colectivos. Incidencia del movimiento indígena en la ley que crea el espacio costero marino de los pueblos originarios. En Fernández, M. y Salinas, J. (Eds.), La defensa de los derechos territoriales desde las comunidades locales en Chile y Latinoamérica: Experiencias y lecciones. Santiago: Programa Ciudadanía y Gestión Pública, CIC, ULA, RIL Editores.

Delamaza, G. y Ochsenius, C. (2010). Innovaciones en los vínculos entre sociedad civil y estado en Chile: $\mathrm{Su}$ incidencia en la construcción de gobernabilidad democrática. Politica y Gestión, 12, 11-35.

Delamaza, G., Maillet, A. y Martínez, C. (2017). Socio-territorial conflicts in Chile: Configuration and politicization (2005-2014). European Review of Latin American and Caribbean Studies/Revista Europea de Estudios Latinoamericanos y del Caribe, 104, 23-46. 
Díaz-Tendero, E. (2014). El derecho de acceso a la información pública como principio de acción en la modernización de la gestión pública y del Estado: Mediciones y efectos en Chile. Revista Transparencia y Sociedad, 1, 37-49.

Durston, J. (2012). Clientelismo político y actores populares en tres regiones de Chile. En Delamaza, G, Cunill, N. y Joignant, A. (Eds.), Nueva agenda de descentralización para Chile. Sentando más actores a la mesa. Santiago: RIL, Universidad de Los Lagos.

El Mostrador. (2019). Los datos finales de la histórica consulta ciudadana organizada por la Asociación Chilena de Municipalidades. 26 de diciembre. Disponible en https://www.elmostrador.cl/noticias/ pais/2019/12/26/los-datos-finales-de-la-historica-consulta-ciudadanaorganizada-por-la-asociacion-chilena-de-municipalidades/ [20-032020].

Foster, B. (2010) Estudio sobre el estado del arte de la investigación sobre participación ciudadana en Chile. Santiago: CISPO.

Fundación para la Superación de la Pobreza (FSP) (2009). Umbrales sociales para Chile. Hacia una futura política social. Santiago: FSP.

. (2013). Umbrales sociales para Chile. Una nueva mirada sobre la pobreza. Santiago: FSP.

Garretón, M. A, (Coord.) (2017). La gran ruptura. Institucionalidad politica y actores sociales en el Chile del siglo XXI. Santiago: LOM.

Instituto Nacional de Derechos Humanos (INDH) (2015). Mapa de conflictos socio-ambientales en Chile. Santiago: INDH. Disponible en http://mapaconflictos.indh.cl/ [31-03-2020].

Lissidini, A. (2015). Democracia directa en América Latina: avances, contradicciones y desafíos. En Minnaert, A. y Endara, G. (Coords.), Democracia participativa e izquierda. Logros, contradicciones y desafíos. Quito: Friedrich-Ebert-Stiftung.

Montecinos, E. (2007). Los límites de la modernización municipal para profundizar la participación ciudadana en Chile ¿Es la gobernanza de proximidad una alternativa? Gestión y Política Pública 16 (2), 319-351. 
Organización para la Cooperación y el Desarrollo Económicos (OCDE) (2017). Chile. La participación ciudadana en el proceso constituyente. Paris: OCDE.

Peña, C. (2020). Pensar el malestar. La crisis de octubre y la cuestión institucional. Santiago: Taurus.

Programa de Naciones Unidas para el Desarrollo (PNUD) (2000). Más sociedad para gobernar el futuro. Santiago: PNUD.

- (2014). Auditoría de la democracia. Más y mejor democracia para un Chile inclusivo. Santiago: PNUD.

. (2015). ¿Qué es el desarrollo humano? Disponible en http://hdr.undp.org/en/node/2228 [03-03-2020].

Poduje, I. (2007) Participación ciudadana en proyectos de infraestructura y planes reguladores. Santiago: Pontificia Universidad Católica de Chile, Vicerrectoría de Comunicaciones y Asuntos Públicos.

Robles, C. (2013) Sistemas de protección social en América Latina y el Caribe: Chile. Santiago: CEPAL.

Ruiz, C. (2017). De nuevo la sociedad. Santiago: LOM Ediciones.

Sociedad en Acción (2017). Sociedad en acción. Construyendo Chile desde las organizaciones de la sociedad civil. Santiago: Centro de Políticas Públicas UC / Chile + Hoy.

Viveros, F. (2018) Modificaciones a la legislación sobre juntas de vecinos y demás organizaciones comunitarias. En Biblioteca del Congreso Nacional (2018), Juntas de vecinos en Chile. 50 años. Historia y desafíos de participación. Santiago: Biblioteca del Congreso Nacional.

Recibido: 25-03-2019

Aceptación de la versión final: 29-12-2019 